Original article

\title{
The basic reproduction number of the new coronavirus pandemic with mortality for India, the Syrian Arab Republic, the United States, Yemen, China, France, Nigeria and Russia with different rate of cases
}

\author{
Marwan Al-Raeei \\ Faculty of Sciences, Damascus University, Damascus, Syrian Arab Republic
}

\section{A R T I C L E I N F O}

\section{Keywords:}

The basic reproduction number COVID-19

SIRD Epidemiology model

Runge-Kutta method

New coronavirus disease

\begin{abstract}
A B S T R A C T
Background: The basic reproduction number values give an initial prediction of the disease because the values predict of end of the disease if the values are less than one or the disease converts to epidemic if the values are more than one. We apply the SIRD epidemiology model for estimating the basic reproduction number of the new coronavirus disease for multiple different countries.

Methods: For estimating of the basic reproduction number values, we fit the SIRD model using the Runge-Kutta simulation method in addition to the analytical solution of parts of the model. We use the collected data of the new coronavirus pandemic reported up to date July 30, 2020 in India, the Syrian Arab Republic, the United States, France, Nigeria, Yemen, China and Russia.

Results: We find that the basic reproduction numbers of the new coronavirus disease are located in the range [1.0011-2.7936] for the different location countries and the values of the ratio between the rate of recovery and the rate of mortality are between 1.5905 for Yemen and 44.0805 for Russia. Also, we find the dates of the actual decreasing of Covid-19 cases in five countries.

Conclusions: We find that the basic reproductive number is between 1.0011 for the smallest value and 2.7936 for the greatest value. The most important thing is that the values of the basic reproduction number of the new coronavirus disease in all considered countries are more than one which means that the new coronavirus disease is epidemic in all of considered countries.
\end{abstract}

\section{Introduction}

The basic reproduction number is very important quantity when we discuss the spreading of a certain disease because it represents the expected cases which is generated by one infectious case in a certain population with a specific pandemic and the basic reproduction number value is very important tool with any pandemic because it predicts that the disease is relaxed when the value is less than one or change to epidemic when the value is more than one. There are multiple method for estimating the basic reproduction number value and all methods depend on the epidemiology models, in this work we use SIRD model for purpose of estimating the basic reproduction number. ${ }^{1-8}$ The SIRD model is described by four differential equations, and these equations describe the change of the susceptible cases $S(t)$, the change of the infectious cases $I(t)$, the change of the recovered cases $R(t)$ and the change of the deceased cases $D(t)$ with respect to the time. The four equations of the SIRD model are given as follows: $\frac{d S(t)}{d t}=-\frac{\alpha_{1}}{N} I(t) S(t)$

$\frac{d I(t)}{d t}=\frac{\alpha_{1}}{N} I(t) S(t)-\alpha_{2} I(t)-\alpha_{3} I(t)$

$\frac{d R(t)}{d t}(t)=\alpha_{2} I(t)$

$\frac{d D(t)}{d t}=\alpha_{3} I(t)$

Where $\alpha_{1}, \alpha_{2}$ and $\alpha_{3}$ are the coefficients of infection, recovery and mortality respectively and $N$ is the population number. We see that all of the four equations contain the population of the infectious cases $I(t)$ because it is the central aspect of the pandemic spreading.

In the following, we apply the previous epidemic model for determining of the basic reproduction number or the basic reproductive ratio

E-mail addresses: mhdm-ra@scs-net.org, mn41@live.com. 
in case of eight countries, namely, the United States where there are the most cases of the pandemic, Russia, China where the first case appeared, India, France, Yemen, Nigeria and the Syrian Arab Republic where we used the collected data of the cases of the new coronavirus pandemic from the references 9-15. In section 2, we illustrate the used method and in section 3, we illustrate the results of the basic reproduction number of the new coronavirus pandemic while in section 4 , we illustrate a conclusion of the study.

\section{The method}

The basic reproduction number for a specific epidemic model can be derived based on multiple methods. Here, we illustrate the method that depends on the eigenvalues of the Jacobian of the differential equation system of the SIRD model. We start by writing the Jacobian as follows:

$J(S, I, R, D)=\left[\begin{array}{cccc}-\frac{\alpha_{1}}{N} I & -\frac{\alpha_{1}}{N} S & 0 & 0 \\ \frac{\alpha_{1}}{N} I & \frac{\alpha_{1}}{N} S-\left(\alpha_{2}+\alpha_{3}\right) & 0 & 0 \\ 0 & \alpha_{2} & 0 & 0 \\ 0 & \alpha_{3} & 0 & 0\end{array}\right]$

Now, we see that the free equilibrium of the pandemic occurs at:

$J_{0}=\left[\begin{array}{cccc}0 & -\alpha_{1} & 0 & 0 \\ 0 & \alpha_{1}-\alpha_{2}-\alpha_{3} & 0 & 0 \\ 0 & \alpha_{2} & 0 & 0 \\ 0 & \alpha_{3} & 0 & 0\end{array}\right]$

By finding the eigenvalues of the Jacobean at the free equilibrium via the following equation:

$\operatorname{det}\left(J_{0}-\lambda \eta_{4}\right)=0$

We find that the eigenvalues are governed by the following algebraic equation of the fourth order degree:

$-\alpha_{1} \lambda^{3}+\alpha_{2} \lambda^{3}+\lambda^{4}+\alpha_{3} \lambda^{3}=0$

By solving the previous equation, we find the following eigenvalues of the Jacobein at the free equilibrium:

$\lambda_{1,2,3}=0$

$\lambda_{4}=\alpha_{1}-\left(\alpha_{2}+\alpha_{3}\right)$

If we take the nontrivial eigenvalue, we find the stable equilibrium and the non-stable equilibrium respectively occur at:

$\alpha_{1}-\left(\alpha_{2}+\alpha_{3}\right)<0$

$\alpha_{1}-\left(\alpha_{2}+\alpha_{3}\right)>0$

Which gives the basic reproduction number from the threshold as follows:

$R_{0}=\frac{\alpha_{1}}{\alpha_{2}+\alpha_{3}}$

As we see to find the basic reproduction number, we need to find the coefficients of the SIRD model. For this purpose, we use analytical solution of Eqs. (3) and (4) of the SIRD model as in the study of the reference ${ }^{1}$ and the Runge-Kutta method for Eqs (1) and (2) of the SIRD model for demonstrating the coefficient of infection, the coefficient of recovery and the coefficient of mortality of the Covid-19 pandemic by fitting the collected cases in eight countries: the Syrian Arab Republic, China, Yemen, India, the united States, France, Nigeria and Russia. Then, based on the values of the coefficients, we find the basic reproduction number of the new corona virus disease in each country. Also, we find the ratio between the recovery rate and the death rate which is important for the difference between the two. Besides, we compute the
Table 1

The coefficient of infection, the coefficient of recovery and the coefficient of mortality of the Covid-19 pandemic for China, the United States, Russia, the Syrian Arab Republic, India, France, Nigeria and Yemen using the reported cases of the pandemic as of July 30, 2020.

\begin{tabular}{llll}
\hline & $\alpha_{1}\left(d^{-1}\right)$ & $\alpha_{2}\left(d^{-1}\right)$ & $\alpha_{3}\left(d^{-1}\right)$ \\
\hline China & 0.0019 & 0.0008 & 0.0003 \\
The United States & 0.0325 & 0.0196 & 0.0005 \\
Russia & 0.0385 & 0.0291 & 0.0007 \\
The Syrian Arab Republic & 0.0625 & 0.0119 & 0.0044 \\
India & 0.1278 & 0.0981 & 0.0037 \\
France & 0.0107 & 0.0034 & 0.0005 \\
Nigeria & 0.0306 & 0.0293 & 0.0013 \\
Yemen & 0.0643 & 0.0281 & 0.0177 \\
\hline
\end{tabular}

Table 2

The basic reproduction number and the ratio between the recovery and the mortality rates of the new coronavirus disease in India, China, the United State, Russia, the Syrian Arab Republic, France, Nigeria and Yemen.

\begin{tabular}{lll}
\hline & $R_{0}$ & $\chi$ \\
\hline India & 1.2561 & 26.8487 \\
China & 1.7273 & 2.7933 \\
The United States & 1.6135 & 37.3383 \\
Russia & 1.2952 & 44.0805 \\
The Syrian Arab Republic & 2.7936 & 2.7000 \\
France & 2.7248 & 6.4267 \\
Nigeria & 1.0011 & 23.1481 \\
Yemen & 1.4067 & 1.5905 \\
\hline
\end{tabular}

average time when the infection may clearly decrease for five countries based on the same simulation method.

\section{Results}

We use the reported data of the collected cases of the new coronavirus disease in the Syrian Arab Republic, China, Yemen, India, the united States, France, Nigeria and Russia up to date July 30, 2020 for finding the coefficient of infection, the coefficient of recovery and the coefficient of mortality of the SIRD model. The coefficients of the SIRD model are illustrated in Table 1 with the units of days ${ }^{-1}$. Then and depending on the previous coefficients, we calculate the basic reproduction number $R_{O}$ of the new corona virus pandemic in the Syrian Arab Republic, China, Yemen, India, the united States, France, Nigeria and Russia. We inserted the results of the basic reproduction number in Table 2 with the ratio between the recovery and the mortality rates for each country which is found by applying the analytical part of the solutions.

Finally, we find the predicted dates of the actual decreasing of the infection cases of the new coronavirus pandemic based on the used model in the United States, China, Russia, India and the Syrian Arab Republic. We illustrate the predicted dates of the actual decreasing of the infection cases in Table 3 for each country.

As we can see from the second table, the greatest values of the basic reproduction number is for the Syrian Arab Republic and the smallest value is for Nigeria which can be explained by the relative infection cases to the recovery and the mortality cases in each country. Also, we can see from the second table that the basic reproduction numbers based on the SIRD model are located between the value 1.5905 for Yemen and 44.0805 for Russia. Besides, if we look at Table 3, we see that the predicted dates for reaching the peak in India, the United States and the Syrian Arab Republic are from August to September while from July to August in Russia. 
Table 3

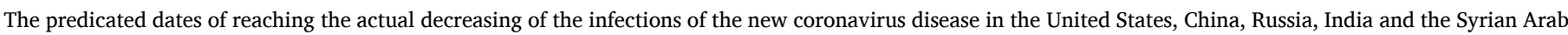
Republic.

\begin{tabular}{|c|c|c|c|c|c|}
\hline & China & The United States & Russia & The Syrian Arab Republic & India \\
\hline The predicted date & Feb-Mar & August-September & July-August & August-September & August-September \\
\hline
\end{tabular}

\section{Conclusion}

In this work, we found the basic reproduction number of the new coronavirus disease for eight countries, namely, Yemen, Nigeria, France, India, China, the United States, Russia and the Syrian Arab Republic (Table 2) based on applying the SIRD model on Covid-19 pandemic in the eight countries. We used the semi analytical method for demonstrating the basic reproduction number of the pandemic by the SIRD model. Besides, we calculated the ratio between the recovery rate and the mortality rate (Table 2) for each country. In addition to that and based on the used model, we calculated the predicted dates of the reaching of the actual decreasing of infectious cases of the new corona virus disease (Table 3) for the United States, China, Russia, India and the Syrian Arab Republic.

We found that the basic reproduction number values of the new coronavirus pandemic are located in the interval [1.0011-2.7936] for the eight countries based on the SIRD model and the smallest values of the basic reproduction number is for Nigeria and the greatest value is for the Syrian Arab Republic which is high relatively value. Also, we found that the actual decreasing of the epidemic in the United States, India and the Syrian Arab Republic may occur from August to September with high values of the infection and the strategy which may be followed to reduce that is increasing the governmental procedures and increasing the treatment which may give more preferable scenario.

\section{Declarations:Availability of data and materials}

The author confirm that the data available for non-commercial using.

\section{Funding}

Not applicable.

\section{Authors' contributions}

Not applicable.

\section{Declaration of competing interest}

The author have no conflict of interests.

\section{Acknowledgments}

Not applicable.

\section{References}

1 Al-Raeei M. The forecasting of COVID-19 with mortality using SIRD epidemic model for the United States, Russia, China, and the Syrian Arab Republic. AIP Adv. 2020;10, 065325.

2 Zhu Peican, et al. Investigation of epidemic spreading process on multiplex networks by incorporating fatal properties. Appl Math Comput. 2019;359:512-524.

3 Kamara Abdul A, et al. Analytical solution for post-death transmission model of Ebola epidemics. Appl Math Comput. 2020;367:124776.

4 Al-Raeei M. Using Methods of Statistical Mechanics in the Study of Soft Condensed Matter Materials and Complex Structures. Damascus, Syrian Arab Republic. Master thesis. 2018.

5 'Izzati Hamdan Nur, et al. A fractional order SIR epidemic model for dengue transmission. Chaos, Solit Fractals. 2018;114:55-62.

6 Osemwinyen Amenaghawon C, et al. Mathematical modelling of the transmission dynamics of ebola virus. Appl Comput Math. 2015;4:313-320.

7 Nathaniel S. Barlow at al (2020) Accurate closed-form solution of the SIR epidemic model. Physica D, 408, 132540.

8 kermack WO. A contirbution to the mathematical theory of epedimic. Proc Roy Soc Lond. 1927;115:700-721.

9 https://www.rosminzdrav.ru/.

10 https://www.moh.gov.sy/.

11 https://www.nhc.gov.cn/.

12 https://www.coronavirus.jhu.edu/.

13 https://www.mohfw.gov.in/.

14 https://www.santepubliquefrance.fr/.

15 https://www.covid-19.ncdc.gov.ng/. 